\title{
Clinical Effects of Planned Endovascular Therapy for Critical Limb Ischemia Patients with Tissue Loss
}

\author{
Masashi Fukunaga, Daizo Kawasaki, Machiko Nishimura, Masanao Yamagami, Reiko Fujiwara and \\ Tsuyoshi Nakata
}

Cardiovascular Division, Morinomiya Hospital, Osaka, Japan

Aim: The aim of this study was to investigate the clinical effect of planned endovascular therapy (EVT) for critical limb ischemia (CLI) patients with tissue loss. Although several rounds of EVT for CLI patients are required for complete wound healing, time required for complete wound healing depends on the wound severity. We hypothesized that planned EVT might reduce the time to wound healing.

Methods: A total of 89 limbs of 76 CLI patients with tissue loss, who had undergone more than at least two EVTs were included in this study. From January 2013 through December 2015 (Conventional-EVT-group, 52 limbs), indication of target lesion revascularization (TLR) was decided based on decreased skin perfusion pressure (SPP) values or delayed wound healing. From January 2016 through October 2016 (Planned-EVT-group, 37 limbs), TLR were done every two months regardless of the SPP values until complete wound healing was obtained. Time to wound healing and complete wound healing rates were compared between the two groups.

Results: No significant differences existed in baseline patients and lesion characteristics between the two groups. There was no significant difference in total EVT numbers between the two groups (2.0; interquartile range, 2.03.0 versus 2.0 ; interquartile range, $2.0-3.0 ; P=0.9)$. Although complete wound healing rate was similar in both groups $(71.2 \%$ versus $73.0 \%, p=1.0)$, time to wound healing was significantly shorter ( 95 days versus 143 days, $p=0.025)$ in the Planned-EVT-group than in the Conventional-EVT-group.

Conclusions: Planned-EVT is a useful strategy to shorten the time to wound healing for CLI patients with tissue loss.

Key words: Catheterization, Critical limb ischemia, Target lesion revascularization (repeat intervention), Wound healing

\section{Introduction}

Critical limb ischemia (CLI) is a condition that represents the most advanced form of peripheral artery disease. Endovascular treatment (EVT) has recently become commonplace as a treatment strategy for CLI ${ }^{1,2)}$. This technique is particularly attractive for patients who are potential candidates for surgery. Iida et al. reported that there was a high rate of reintervention in CLI patients and 25\% amputations were observed from the multicenter registry ${ }^{3}$. Although several rounds of EVT in CLI patients with tissue loss (Rutherford category 5 or 6) were required until complete wound healing was attained, time to wound healing depended on the wound severity. Early recurrence after EVT and prolonged wound healing make CLI management cumbersome. In general, timing of target lesion revascularization (TLR) is planned when wound healing is delayed and/ or there is suspected recurrence of treated vessels with decreased skin perfusion pressure (SPP). This approach leads to the dual problems of potential prolonged wound healing and delayed rehabilitation. We hypothesized that planned EVT before complete recurrence might shorten the time to wound healing.

Address for correspondence: Masashi Fukunaga, Cardiovascular Division, Morinomiya Hospital, 2-1-88 Morinomiya Joto-ku, Osaka, Osaka, 536-0025, Japan 


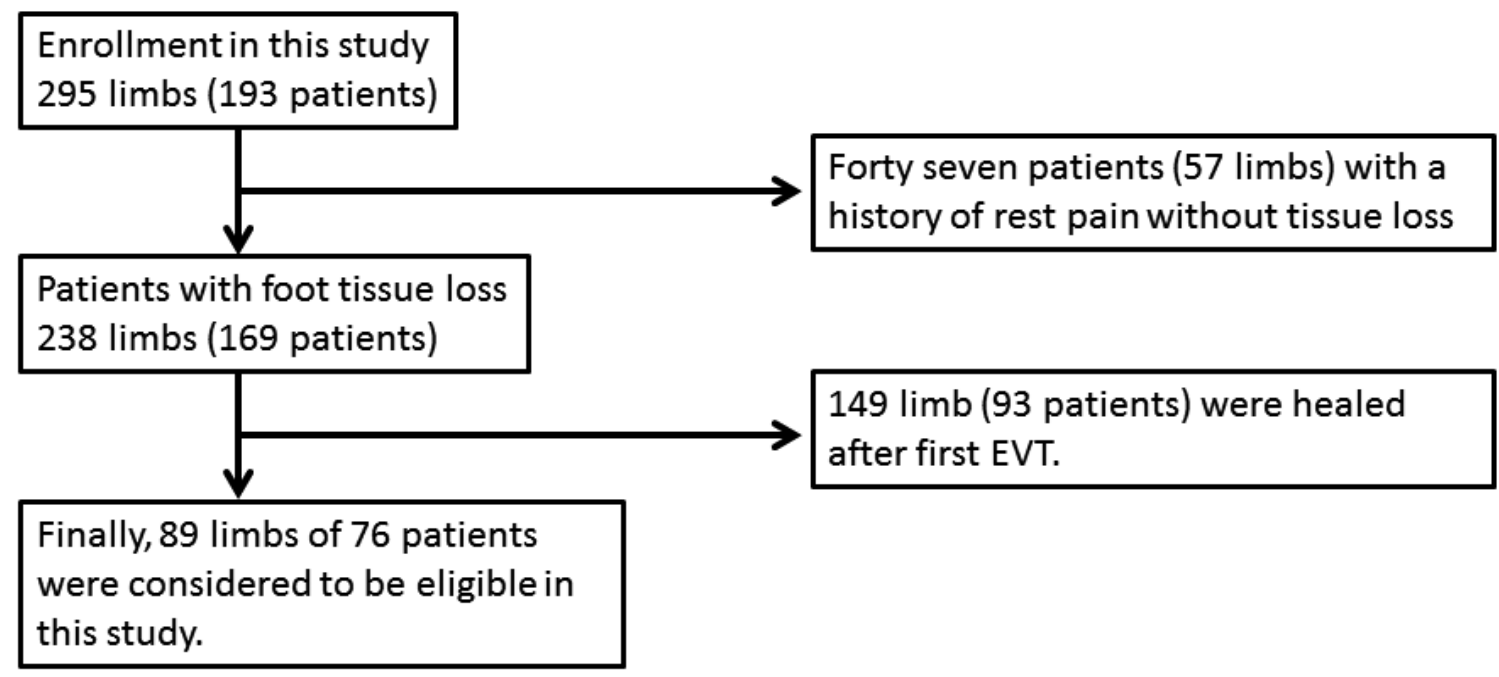

Fig. 1. Flow diagram of this study.

\section{Aim}

The aim of this study was to investigate the clinical utility of planned EVT in CLI patients with tissue loss.

\section{Methods}

Study Design and Population

Between January 2013 and October 2016, 244 limbs (175 CLI patients) suffered from non-healing ulcers or gangrene. Six patients $(3.4 \%)$ were treated with surgical bypass graft; then a total of 238 limbs (169 CLI patients) with non-healing ulcers or gangrene classified as Rutherford 5 or 6, underwent EVT as the primary treatment at our institution. 149 limbs (93 patients) in which complete wound healing was attained with only the first EVT were excluded. Finally, a total of 89 limbs of 76 CLI patients with tissue loss who underwent EVT more than at least two times were included in this study (Fig. 1).

From January 2013 through December 2015 (Conventional EVT group, 52 limbs), timing of TLR was decided based on delayed wound healing accompanied by decreased SPP values. A SPP value less than 40 $\mathrm{mmHg}$ was defined as evidence of insufficient flow to the wound. From January 2016 through October 2016 (Planned EVT group, 37 limbs), TLRs were done every two months regardless of the SPP values, until complete wound healing was attained (Fig. 2).

Endovascular Treatment and Angiographic Analysis

Whole lower limb artery (abdominal artery to foot artery) was routinely evaluated by duplex ultrasound and/or CT angiogram. Ischemic severity of the wound was hemodynamically assessed by using SPP value. Angiography was performed before and immediately after EVT. Quantitative vascular angiography analysis using commercially available software (CAAS 5.9, Pie Medical Imaging, Maastricht, The Netherlands) was performed in a blinded manner to determine lesion severity and to evaluate the degree of residual stenosis in the artery before EVT. The tip of the guiding catheter was used for calibration in the analysis because movement of the catheterization table was required for angiographic evaluation of whole infrapopliteal lesions. Therefore, the diameters of the reference vessel, lumen, and lesion length were impossible to calculate, and only the percent diameter stenosis was calculated as follows: minimum lumen diameter divided by the average ([proximal + distal $] \times 1 / 2)$ reference vessel diameter. EVT was conducted when the lesion had diameter stenosis more than $75 \%$ on digital subtraction angiography (DSA). If the patients had aorto-iliac lesions and/or femoropopliteal lesions, we treated them with EVT before BTK interventions. All EVT procedures were generally attempted based on the angiosome concept ${ }^{9)}$ and the angiosome-based favorable target lesion was confirmed by DSA. Additional interventions for non-angiosomebased lesions were attempted if DSA showed a severe stenosis and/or occlusion in the non-target artery. The antegrade approach from the ipsilateral common femoral artery was selected for all patients. After insertion of a 4.5 Fr guiding sheath (Parent Plus 4.5, Medikit, Tokyo, Japan), unfractionated heparin (100 units $/ \mathrm{kg}$ ) was administered into the artery. Anticoagulation was accomplished by maintaining the activated clotting time within the range of 250-300 s during the procedure. 


\section{Phase 1 ; Conventional EVT}

Delayed wound healing and/or decrease SPP values

\section{1}

2016.1

2016.10

\section{Phase 2 ;Planned EVT}

EVT before decreasing SPP values

Fig. 2. Study design.

Phase 1; From January 2013 through December 2015, timing of TLR was decided based on delayed wound healing accompanied by decreased SPP values. A SPP value less than $40 \mathrm{mmHg}$ was defined as evidence of insufficient flow to the wound.

Phase 2; From January 2016 through October 2016, TLRs were done every two months regardless of the SPP values, until complete wound healing was obtained.

Dual anti-platelet therapy (DAPT; aspirin at $100 \mathrm{mg} /$ day and cilostazol at $200 \mathrm{mg} /$ day, or clopidogrel at 75 $\mathrm{mg} /$ day) was initiated at least 1 week prior to EVT.

After a 0.014/0.018-inch guidewire was introduced into the lesion, balloon angioplasty was performed by using a conventional balloon with a diameter equal to the reference vessel diameter according to visual estimation. Balloon inflation was held for a total $90 \mathrm{~s}$ with nominal pressure. If the residual stenosis was more than $30 \%$, inflation times were prolonged to 180 s. Procedural success was defined when the residual diameter stenosis was $\leq 30 \%$ on angiogram. After EVT, DAPT was prescribed to all the patients for at least 3 months.

\section{Timing of Target Lesion Revascularization}

Conventional EVT groups; TLR was conducted when wound healing was delayed accompanied by decreased SPP values.

Planned EVT groups; TLR was conducted after two months of each EVT regardless of the SPP values until complete wound healing was attained.

\section{Ulcer Assessment}

Before EVT, lesions were photographed in patients with ulcers or gangrene from three different angles with a control-colored measuring tape applied near the wound. Ulcers were photographed from the same angles at each subsequent outpatient follow-up to monitor the healing process. The assessor also determined the presence or absence of infection. All wounds were treated by an experienced plastic surgeon and cardiologist.

\section{Primary End Points}

Time to wound healing and complete wound healing rate were analyzed between the two groups.

\section{Statistical Analysis}

Continuous data were expressed as mean \pm standard deviation, or median and categorical data were expressed as numbers with percentages. Continuous variables were compared using the $t$ test or MannWhitney $U$ test, as appropriate. Categorical variables were compared by the maximum likelihood chi-square test or Fisher exact test. Survival curves were estimated using the Kaplan-Meier method and compared using the univariate log-rank test. All statistical analyses were performed with EZR (Saitama Medical Center, Jichi Medical University, Shimotsuke, Tochigi, Japan), which is a graphical user interface for $\mathrm{R}$ (version 2.13.0; The $\mathrm{R}$ Foundation for Statistical Computing, Vienna, Austria). More precisely, it is a modified version of $\mathrm{R}$ commander (version 1.8-4) designed to add statistical functions frequently used in biostatistics.

\section{Results}

All procedures were successfully completed without any complications. 52 limbs (43 patients) were assigned to the conventional EVT group and 37 limbs (33 patients) were assigned to the planned EVT group. The patients' baseline characteristics, limb characteristics, and angiographic findings are presented in Tables 1,2 , and 3. No significant differences existed in patients' 
Table 1. Baseline Clinical Characteristics

\begin{tabular}{lccc}
\hline & $\begin{array}{c}\text { Conventional-EVT } \\
(n=43)\end{array}$ & $\begin{array}{c}\text { Planned-EVT } \\
(n=33)\end{array}$ & $p$ value \\
\hline Age, yrs & $73 \pm 9$ & $73 \pm 12$ & 0.85 \\
Body mass index & $21.2 \pm 3.1$ & $21.1 \pm 3.7$ & 0.79 \\
Male gender, \% & $27(62)$ & $24(73)$ & 0.82 \\
Hypertension, \% & $30(70)$ & $28(85)$ & 0.59 \\
Dyslipidemia, \% & $19(44)$ & $16(48)$ & 1.00 \\
Diabetes mellitus, \% & $26(60)$ & $24(73)$ & 0.81 \\
Current smoker, \% & $18(42)$ & $17(52)$ & 0.82 \\
Hemodialysis, \% & $26(60)$ & $26(79)$ & 0.33 \\
Medication & & $21(64)$ & 0.36 \\
$\quad$ Aspirin, \% & $20(47)$ & $16(48)$ & 0.25 \\
$\quad$ Clopidogrel, \% & $24(56)$ & $12(36)$ & 0.36 \\
$\quad$ Cilostazol, \% & $20(47)$ & $5(15)$ & 1.00 \\
$\quad$ Anticoagulant, \% & $7(16)$ & $3.58 \pm 0.45$ & 0.28 \\
Laboratory date & & $3.29 \pm 4.91$ & 0.04 \\
$\quad$ Serum albumin, mg/dl & $6(18)$ & 0.58 \\
$\quad$ CRP, mg/dl & $3.47 \pm 0.48$ & & \\
Nonambulatory, \% & $1.67 \pm 2.37$ & $11(26)$ &
\end{tabular}

Values are given as $\mathrm{n}(\%)$ or mean \pm standard deviation.

Table 2. Limb Characteristics

\begin{tabular}{|c|c|c|c|}
\hline & $\begin{array}{l}\text { Conventional-EVT } \\
\qquad(n=52)\end{array}$ & $\begin{array}{l}\text { Planned-EVT } \\
\quad(n=37)\end{array}$ & $p$ value \\
\hline Right/Left & $21(40) / 31(60)$ & $21(57) / 16(43)$ & 0.14 \\
\hline Rutherford class $5 / 6$ & $43(83) / 9(17)$ & $31(84) / 6(16)$ & 1.0 \\
\hline \multicolumn{4}{|l|}{ Ulcer Location } \\
\hline Toe & $49(94)$ & $35(95)$ & 1.0 \\
\hline Dorsum & $11(21)$ & $9(24)$ & 0.80 \\
\hline Planter & $11(21)$ & $8(22)$ & 1.0 \\
\hline Heel & $5(10)$ & $3(8)$ & 1.0 \\
\hline Wound infection & $36(69)$ & $18(49)$ & 0.078 \\
\hline Minor amputation & $18(35)$ & $16(43)$ & 0.51 \\
\hline Major amputation & $3(6)$ & $2(5)$ & 1.0 \\
\hline Clinical stage assessed by WIfI & & & 0.399 \\
\hline $1(\%)$ & $4(7.7)$ & $6(16.2)$ & \\
\hline $2(\%)$ & $9(17.3)$ & $3(8.1)$ & \\
\hline $3(\%)$ & $18(34.6)$ & $11(29.7)$ & \\
\hline $4(\%)$ & $21(40.4)$ & $17(45.9)$ & \\
\hline
\end{tabular}

Values are given as $\mathrm{n}(\%)$.

Abbreviations: EVT = Endovascular therapy;

WIfI = the Wound, Ischemia, and foot Infection classification system.

baseline characteristics between the two groups. The most prevalent comorbidities were hypertension $(76 \%$; $58 / 76)$, hemodialysis $(68 \% ; 52 / 76)$, and diabetes mellitus (66\%; 50/76) (Table 1).

Limb characteristics are listed in Table 2. Accord- ing to the Rutherford classification, there were no significant differences between the two groups. Ulcers were located mostly in the toe in both groups and wound infections were present in more than $50 \%$ patients.

Lesion characteristics were similar in both groups. 
Table 3. Lesion Characteristics

\begin{tabular}{|c|c|c|c|}
\hline & $\begin{array}{l}\text { Conventional-EVT } \\
\qquad(n=52)\end{array}$ & $\begin{array}{l}\text { Planned-EVT } \\
\qquad(n=37)\end{array}$ & $p$ value \\
\hline \multicolumn{4}{|l|}{ Culprit lesions } \\
\hline AI plus FP plus BK lesions, (\%) & $10(19.2)$ & $1(2.7)$ & 0.02 \\
\hline FP plus BK lesions, $(\%)$ & $24(46.2)$ & $22(59.5)$ & 0.28 \\
\hline BK lesions, (\%) & $18(34.6)$ & $14(37.8)$ & 0.82 \\
\hline Run off vessels before EVT & & & 0.02 \\
\hline $0 / 1 / 2 / 3$ & $13 / 28 / 11 / 0$ & $17 / 17 / 3 / 0$ & \\
\hline Median Run off vessels before EVT & $1, \mathrm{IQR}, 0.75-1.0$ & 1, IQR, 0-1 & 0.003 \\
\hline Run off vessels after EVT & & & 0.38 \\
\hline $0 / 1 / 2 / 3$ & $0 / 8 / 38 / 6$ & $0 / 10 / 14 / 13$ & 0.90 \\
\hline Median Run off vessels after EVT & 2, IQR, 2-2 & 2, IQR, 1-3 & 0.15 \\
\hline Total EVT number & 2, IQR, 2-3 & 2, IQR, 2-3 & 0.90 \\
\hline BA run off before EVT & $0, \mathrm{IQR}, 0.0-1.0$ & $0, \mathrm{IQR}, 0.0-1.0$ & 0.754 \\
\hline BA run off after EVT & $0, \mathrm{IQR}, 1.0-2.0$ & $0, \mathrm{IQR}, 1.0-2.0$ & 0.716 \\
\hline Patent pedal arch before EVT, (\%) & $12(23.1)$ & $10(27)$ & 0.804 \\
\hline Patent pedal arch after EVT, (\%) & $23(44.2)$ & $22(59.5)$ & 0.198 \\
\hline frequency of angiosome-oriented angioplasty, (\%) & $42(80.8)$ & $27(73.0)$ & 0.445 \\
\hline
\end{tabular}

Values are given as mean \pm standard deviation.

Abbreviations: $\mathrm{AI}=$ Aorto-iliac lesions, $\mathrm{BA}=$ Below the ankle lesions, $\mathrm{BK}=$ Below the knee lesions, $\mathrm{EVT}=\mathrm{Endovascular}$ treatment; $\mathrm{FP}=$ Femoropopliteal lesions, IQR; interquartile range

Prevalence of femoro-popliteal lesions plus below the knee (BK) lesions were not significantly different between the two groups (34\% versus 38\%, $p=0.84$ ). Prevalence of isolated BK lesions were also not significantly different between the two groups (46\% versus 59\%, $p=$ 0.28). Median number of runoff vessels before EVTs were fewer in the planned EVT group than in the conventional-EVT-group (1.0, interquartile range [IQR], $0-1.0$ versus $1, \mathrm{IQR}, 0.75-1.00, p=0.02)$. There was no significant difference in median number of runoff vessels after EVTs (2.0, [IQR], 2.0-2.0 versus 2, IQR, $1.0-3.0, p=0.38$ ).

The interval between the first and second EVT in the conventional EVT group was longer than that in the planed EVT group $(78.8 \pm 45.7$ versus $46.1 \pm 16.6$, $p<0.001)$.

Ten cases in the conventional EVT group were treated with EVT within two months because of delayed wound healing or decrease of SPP values. One TLR in the planned EVT group was required within one month because the wound area became worse after 14 days.

\section{Primary End Points}

Sixty-five $(72 \%)$ limbs of all patients attained complete wound healing without major amputation.

Although the rate of wound healing was similar in both groups $(71.2 \%$ versus $73.0 \% ; p=1.0$, Fig. 3 ), time to wound healing was significantly shorter in the conventional EVT group than in the planned EVT group ( $143 \pm 99$ days versus $95 \pm 48$ days; $p=0.0245$, Fig. 4).

There was no statistically significant difference in the total number of EVTs done until complete wound healing was attained between the two groups (2.0, [IQR], $2.0-3.0$ versus 2 , IQR, $2.0-3.0, p=0.90$, respectively) (Table 3).

\section{Discussion}

The main purpose of the present study was to evaluate whether planned EVT can become the standard strategy to manage CLI patients with tissue loss. Planned EVT strategy was useful in reducing wound healing periods vis-à-vis the conventional strategy. To the best of our knowledge, this is the first report to investigate the relationship between planned EVT and time to wound healing.

Our data suggests that wound healing rates were similar in both groups. More than $70 \%$ of limbs treated with repeat interventions in this study attained complete wound healing. In addition, about 213 (89.5\%) limbs with/without repeat interventions obtained complete wound healing in our total study population. Previous studies have reported time to wound healing and wound healing rates after revascularization ${ }^{4-7)}$. After EVT for CLI with tissue loss, a 54-86\% cumulative wound healing rate at 1 year was achieved. 


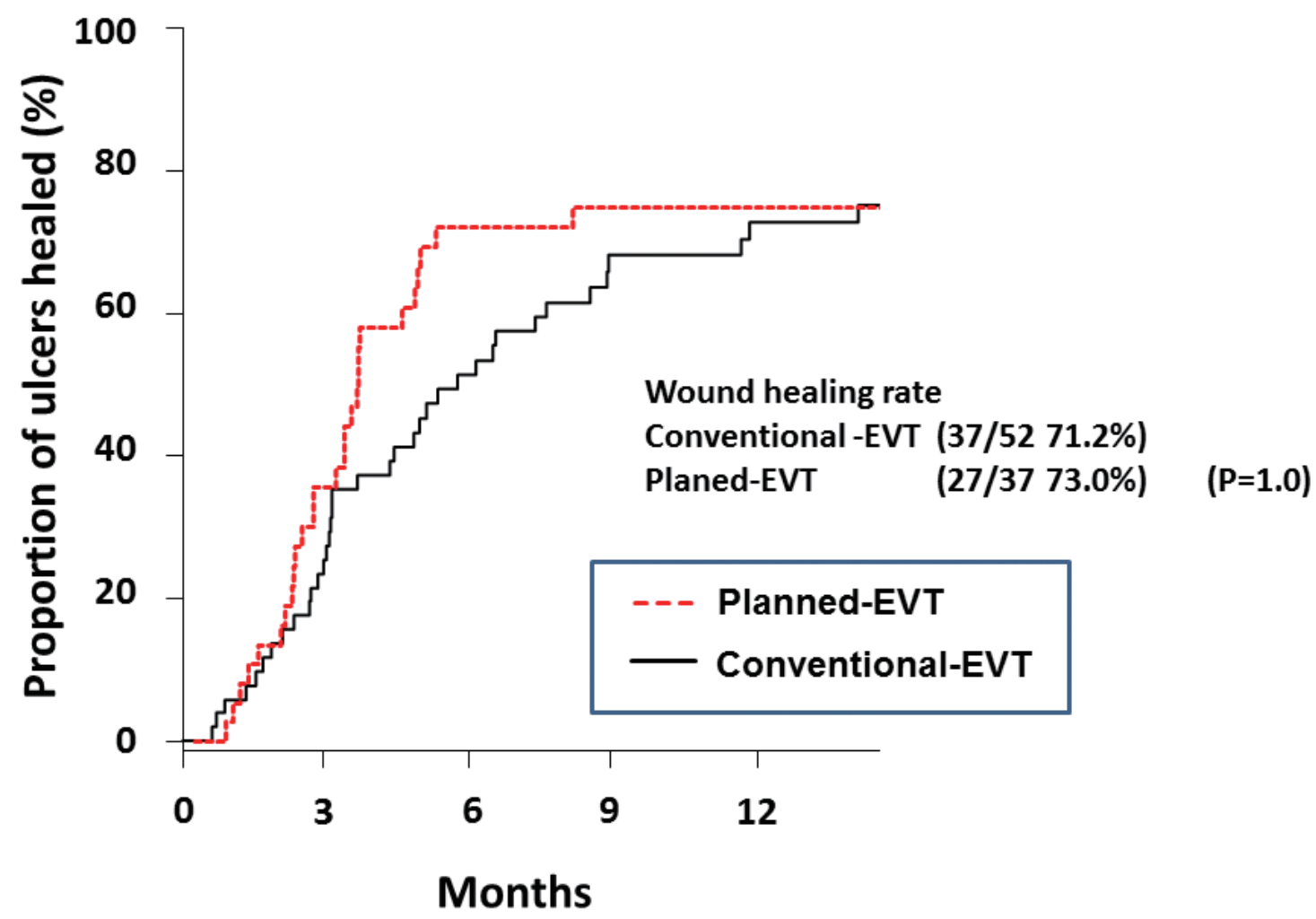

Fig. 3. Wound healing rates at 1 year in Conventional-EVT and Planned-EVT groups.

The rate of wound healing was similar in the both groups $(71.2 \%$ versus $73.0 \%$; $p=1.0)$.

Although revascularization was successful, wound healing was not complete in some of the patients. In previous reports, wound healing was correlated with blood flow volume, infection, and the method of wound management ${ }^{3,8)}$. Castronuovo et al. ${ }^{9)}$ reported that the measurement of SPP was useful for the evaluation of the microcirculatory condition after EVT in patients with CLI and that SPP values $\geq 40 \mathrm{mmHg}$ were associated with wound healing in critically ischemic limbs. SPP was useful in the assessment of microcirculation simplicity and reproducibility after EVTs. When the arterial stenosis or occlusion occurs after EVT, blood flow volume reduces to the wound area. We have previously reported that less vasculature or microvascular damage was a worse predictor of wound healing ${ }^{10)}$.

Wound care without reduction of blood flow is important in achieving complete wound healing. At the point of blood flow, bypass surgery (BS) is a better treatment for patients with CLI. Although the BS versus Angioplasty in Severe Ischemia of the Leg trial showed better long-term amputation-free survival outcomes after BS on autologous veins compared with $\mathrm{EVT}^{11)}$, a major amputation has been reported to be necessary in approximately $10 \%$ of patients with ischemic ulcers of the lower limbs despite a patent bypass ${ }^{12}$.
EVT is now considered the acceptable therapy in CLI attributable to infrapopliteal lesions in some patients.

Ito $\mathrm{R}$ et al. reported the long-term clinical outcome of EVT or BS in patients on hemodialysis (HD) with critical limb ischemia due to isolated infrapopliteal disease. In patients with $\mathrm{HD}$, freedom from any re-intervention was markedly lower in the EVT group than in the bypass group ${ }^{13)}$. On the other hand, Shiraki $\mathrm{T}$ et al. who compared clinical outcomes after BS and after EVT using propensity score matching, concluded that in HD patients with CLI who underwent infrainguinal revascularization, overall survival, major amputation, repeat-EVT, and surgical reconstruction were not significantly different after EVT versus $\mathrm{BS}^{14)}$. In our study population, HD patients were included more than $50 \%$. It was difficult to manage the wound; in addition EVT was also difficult because of vessel calcification and lesion tortuosity.

Delayed wound healing affects quality of life (QOL) in patients with tissue loss and impedes social rehabilitation. Our treatment modality will improve the QOL for these patients. 


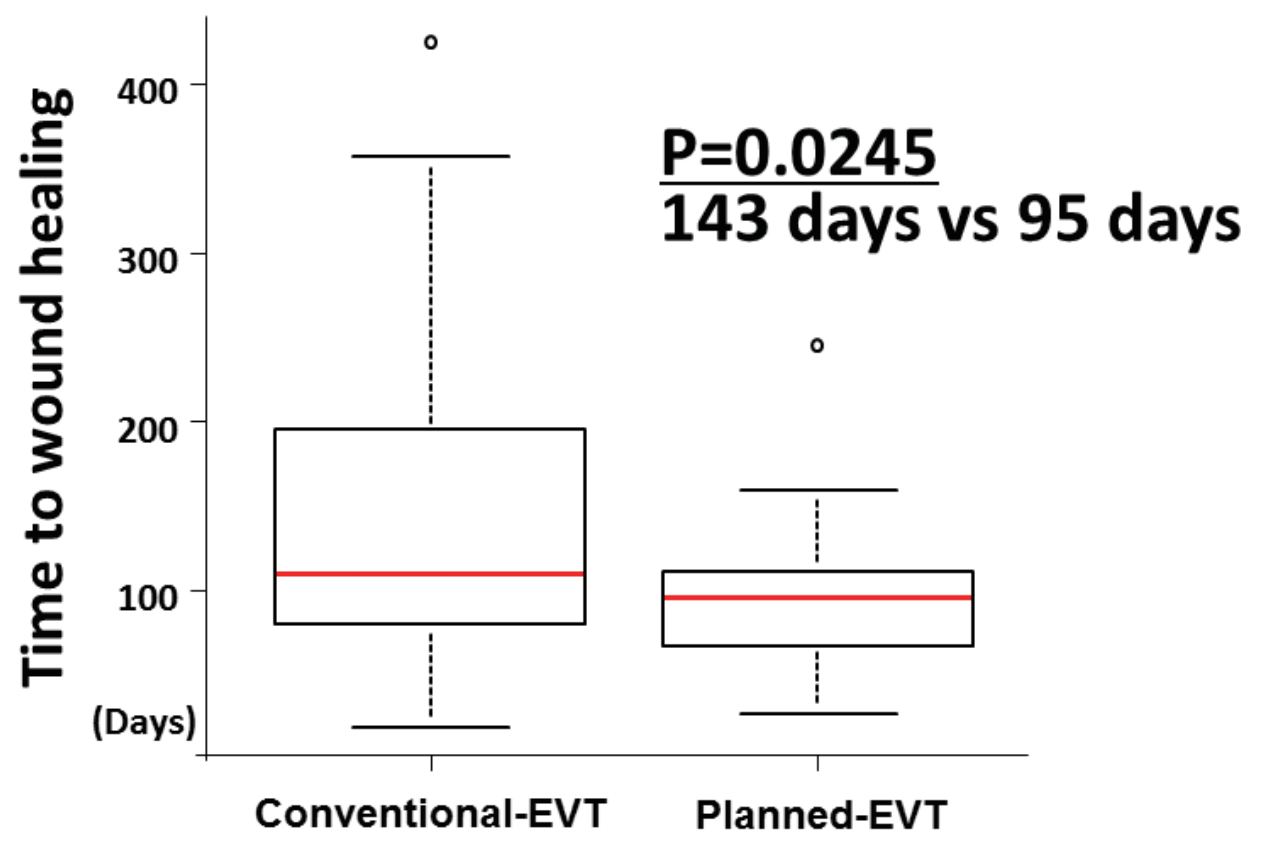

Fig.4. Time to complete wound healing.

Time to wound healing was significantly shorter in the Conventional-EVT group than in the PlannedEVT group ( $143 \pm 99$ days versus $95 \pm 48$ days; $p=0.0245$ ).

\section{Study Limitations}

First, this study was a retrospective, single center, non-randomized analysis and thus the sample size was relatively small. Hence, multicenter studies with large numbers of patients are required to confirm the present results. Second, wound healing was affected by several factors, such as the treatment technique (used) by plastic surgeons, infections, and recurrence.

Third, the angioplasty we used, employed the conventional balloon, and not the drug coated balloon, as drug coated balloons are still not available in japan.

\section{Conclusions}

Planned EVT was useful in reducing time to wound healing in CLI patients with tissue loss. This strategy may become the standard approach in managing patients with tissue loss

\section{Acknowledgments}

The authors thank the staff in the catheterization laboratory at Morinomiya hospital for their excellent assistance during the study.

\section{Disclosures}

None.

\section{References}

1) Dormandy JA, Rutherford RB. Management of peripheral arterial disease (PAD). TASC Working Group. TransAtlantic Inter-Society Consensus (TASC). J Vasc Surg 2000; 31: $1-296$

2) Norgren L, Hiatt WR, Dormandy JA, et al., TASC II Working Group. Inter-Society Consensus for the Management of Peripheral Arterial Disease (TASC II). Eur J Vasc Endovasc Surg 2007; 33 Suppl 1: S1-75

3) Iida O, Nakamura M, Yamauchi $Y$, Kawasaki D, Yokoi $Y$, Yokoi H, Soga Y, Zen K, Hirano K, Suematsu N, Inoue N, Suzuki K, Shintani Y, Miyashita Y, Urasawa K, Kitano I, Yamaoka T, Murakami T, Uesugi M, Tsuchiya T, Shinke T, Oba Y, Ohura N, Hamasaki T, Nanto S; OLIVE Investigators. Endovascular treatment for infrainguinal vessels in patients with critical limb ischemia: OLIVE registry, a prospective, multicenter study in Japan with 12-month follow-up. Circ Cardiovasc Interv. 2013; 6: 68-76

4) Söderstrom M, Aho PS, Lepäntalo M, Albäck A. The influence of the characteristics of ischemic tissue lesions on ulcer healing time after infrainguinal bypass for critical leg ischemia. J Vasc Surg 2009; 49: 932-937

5) Chung J, Bartelson BB, Hiatt WR, Peyton BD, McLafferty RB, Hopley CW, et al.Wound healing and functional outcomes after infrainguinal bypass with reversed saphenous vein for critical limb ischemia. J Vasc Surg 2006; 43: 11831190

6) Azuma N, Uchida H, Kokubo T, Koya A, Akasaka N, Sasajima T. Factors influencing wound healing of critical limb ischemia foot after bypass surgery: is the angiosome important in selecting bypass target artery? Eur J Vasc Endovasc 
Surg 2012; 43: 322-328

7) Rocha-Singh KJ, Jaff M, Joye J, Laird J, Ansel G, Schneider P. Major adverse limb events and wound healing following infrapopliteal artery stent implantation in patients with critical limb ischemia: the XCELL Trial. Catheter Cardiovasc Interv 2012; 80: 1042-1051

8) Kawarada O, Fujihara M, Higashimori A, Yokoi Y, Honda Y, Fitzgerald PJ. Predictors of adverse clinical outcomes after successful infrapopliteal intervention. Catheter Cardiovasc Interv 2012; 80: 861-871

9) Castronuovo JJ Jr, Adera HM, Smiell JM, Price RM. Skin perfusion pressure measurement is valuable in the diagnosis of critical limb ischemia. J Vasc Surg. 1997; 26: 629-637

10) Masashi Fukunaga, Kenichi Fujii, Daizo Kawasaki, Machiko Nishimura, Tetsuo Horimatsu, Ten Saita, Kojiro Miki, Hiroto Tamaru, Takahiro Imanaka, Yoshiro Naito, Tohru Masuyama. Vascular Flow Reserve Immediately After Infrapopliteal Intervention as a Predictor of Wound Healing in Patients With Foot Tissue Loss. Circ Cardiovasc Interv. 2015; 8 DOI https://doi.org/10.1161/CIRCINTERVEN-
TIONS.115.002412

11) BASIL participants. Bypass versus angioplasty in severe ischemia of the leg (BASIL): Multicentre, randomized controlled trial. Lancet. 2005; 366: 1925-1934

12) Berceli SA, Chan AK, Pomposelli FB Jr, Gibbons GW, Campbell DR, Akbari CM, Brophy DT, LoGerfo FW. Efficacy of dorsal pedal artery bypass in limb salvage for ischemic heel ulcers. J Vasc Surg. 1999; 30: 499-508

13) Ito R, Kumada Y, Ishii H, Kamoi D, Sakakibara T, Umemoto N, Takahashi H, Murohara T. Clinical Outcomes after Isolated Infrapopliteal Revascularization in Hemodialysis Patients with Critical Limb Ischemia: Endovascular Therapy versus Bypass Surgery. J Atheroscler Thromb. 2018; 25: 799-807

14) Shiraki T, Iida O, Takahara M, Soga Y, Mii S, Okazaki J, Kuma S, Yamaoka T, Kamoi D, Shintani Y, Ishikawa T, Kitano I, Uematsu M. Comparison of Clinical Outcomes after Surgical and Endovascular Revascularization in Hemodialysis Patients with Critical Limb Ischemia. J Atheroscler Thromb. 2017; 24: 621-629 